\title{
Quantitative Mobility Spectrum Analysis of carriers in GaSb/InAs/GaSb superlattice
}

\author{
T.V. Chandrasekhar Rao ${ }^{a, c}$, J. Antoszewski ${ }^{a}$, J.-B. Rodriguez ${ }^{b}$, E. Plis ${ }^{b}$, S. Krishna ${ }^{b}$, L. \\ Faraone $^{\mathrm{a}}$ \\ ${ }^{a}$ School of Electrical, Electronic and Computer Engineering, The University of Western \\ Australia, Crawley, WA 6009, Australia \\ ${ }^{\mathrm{b}}$ Center for High Technology Materials, Department of Electrical and Computer Engineering, \\ University of New Mexico, Albuquerque, New Mexico 87106
}

\begin{abstract}
We have investigated electrical transport in Type II GaSb/InAs superlattice grown on GaSb, using 'Quantitative Mobility Spectrum Analysis' (QMSA). Our results indicate that the superlattice contributes a lone electron specie with an ambient temperature mobility of $\sim 10^{4}$ $\mathrm{cm}^{2} / \mathrm{Vs}$. Variable temperature studies in the range $50 \mathrm{~K}-300 \mathrm{~K}$ show that the carrier is associated with an activation energy of $0.27 \mathrm{eV}$, which is very close to the superlattice band gap.
\end{abstract}

Material names: InAs, GaSb, superlattice

\footnotetext{
${ }^{c)}$ On leave from Technical Physics and Prototype Engineering Division, Bhabha Atomic Research Centre, Bombay 400085, India. Electronic mail: chandra1 @ barc.gov.in
} 


\section{Introduction:}

Type II GaSb/InAs/GaSb superlattices have shown a great potential as detector materials in midIR range (3-5 $\mu \mathrm{m})$ [1] and are considered a possible alternative to the established mercury cadmium telluride system for device applications [2, 3] with comparable quantum efficiencies [4]. An added advantage is their operability at ambient temperatures, since detection is based on interband optical transitions, requiring less cooling than detectors based on extrinsic silicon. Short period GaSb/InAs superlattices are grown on undoped GaSb substrate using molecular beam epitaxy (MBE) and material performance is critically affected by parameters such as interfacial roughness, residual carrier concentration etc. Therefore it is imperative to investigate electrical transport in these materials with a view to optimize growth conditions and fine tune the device performance.

Electrical characterization of these superlattices is a challenge as GaSb substrate is highly conductive and its contribution to sample conductivity will obscure that of superlattice (substrate being much thicker than superlattice film). There were attempts to circumvent this problem by introducing a lattice matched buffer layer between the substrate and the superlattice, but concerns were raised about its influence on residual superlattice carrier concentration [2]. In order to unravel the nature of conduction within superlattice itself we have used the approach of 'Quantitative Mobility Spectrum Analysis' (QMSA) [5]. This method is based on variable magnetic field resistivity \& Hall Effect measurements and is a versatile tool to analyze electrical transport in multi-carrier and multilayer systems, even if some carriers contribute significantly less to sample conduction than others [6]. Basically, high mobility carriers are 'turned-off' under 
higher magnetic fields, paving a way for differentiating carriers on the basis of their mobility and resolving variable magnetic field conductivity data into contributions from individual carriers. QMSA algorithm converts experimentally measured magnetic field dependent resistivity and Hall coefficient data into 'carrier mobility spectra', showing conductivity of each carrier plotted against its mobility. A cumulative analysis of these spectra provides the mobility and density of all contributing carrier species.

In this report, we present the results of "Quantitative Mobility Spectrum Analysis" (QMSA) of a GaSb/InAs superlattice sample. Our motivation was to check if QMSA can identify and evaluate carriers arising from the superlattice despite a domineering contribution to conductivity from those emanating from the substrate.

\section{Measurements:}

Our measurements were made on a 500-period unintentionally doped superlattice, each period consisting of '9 monolayers of GaSb / 9 monolayers of InAs', grown on a $490 \mu \mathrm{m}$ thick GaSb substrate (undoped, but residually p-type). Total thickness of the superlattice, thus, was approximately $3 \mu \mathrm{m}$. InAs/GaSb material was grown using a solid source VG80 molecular beam epitaxy (MBE) reactor on a $\mathrm{GaSb}(001)$ substrate, using $\mathrm{As}_{2}$ (valved) and $\mathrm{Sb}_{2}$ cracker sources. Growth rates were set respectively to 0.33 and 0.5 monolayers/second for InAs and GaSb. A relatively low growth temperature (about $400^{\circ} \mathrm{C}$ ) ensures a good quality of InSb-like interfaces [1]. 12s Sb and 2s As soak times of InAs and GaSb layers, respectively, were used in order to further sharpened the interfaces. High-resolution X-ray diffraction scan of the sample showed intense and numerous satellite peaks, attesting its good crystalline quality. 
Resistivity and Hall coefficient were measured on a sample piece of area $5 \times 5.5 \mathrm{~mm}^{2}$ using four electrical contacts to the superlattice layer in Van der Pauw configuration, as shown in Fig. 1. Contact pads consisting of $\mathrm{Ti}, \mathrm{Pt}$ and $\mathrm{Au}$ were deposited by thermal evaporation and were found to be Ohmic. Variable magnetic field was provided by a superconducting magnet (manufactured by Oxford Instruments; field range 0 to $\pm 10 \mathrm{~T}$ ) and sample temperature was varied between $50 \mathrm{~K}$ and $300 \mathrm{~K}$ with a liquid helium cryostat. A constant current of $10 \mathrm{~mA}$ was passed through the sample as voltage drops were measured across appropriate pairs of contacts according to standard switching procedures for resistivtity and Hall Effect measurements. To evaluate substrate contribution to sample conduction, separate measurements were made on a piece of substrate (without superlattice), 440 $\mu \mathrm{m}$ thick, under identical measurement conditions.

Data was analyzed by Lakeshore i-QMSA package based on an algorithm outlined earlier [6]. The output of this algorithm is a 'mobility spectrum' (see for example Fig. 2), depicting peaks corresponding to each of the carriers present. Typically, each peak consists of a small distribution of mobilities and the corresponding contribution to sample conductivity. Carrier density and mobility associated with each peak can be evaluated, respectively, by summing up number densities from each of the spectral channels and by taking a mean of the associated mobility range:

$$
n_{i}=\sum_{j=1}^{N} \frac{\sigma_{\mathrm{j}}}{q \mu_{j}} \quad \text { and } \quad \mu_{i}=\frac{1}{N}\left(\sum_{j=1}^{N} \mu_{j}\right)
$$

where $n_{i}$ and $\mu_{i}$ are the density and mobility of $i^{\text {th }}$ carrier and $q$ is the electron charge. 


\section{Results and Discussion:}

We have noted that the Hall coefficient for the sample was positive in the entire temperature range investigated $(50 \mathrm{~K}-300 \mathrm{~K})$, indicating hole dominance of conductivity, presumably from the substrate [7]. QMSA fits to experimental data were excellent for all temperatures. Fig. 2 depicts a typical mobility spectrum from the sample (superlattice + substrate), showing five carrier species in all - three electrons and two holes. These have been labeled as $\mathrm{e}_{1}, \mathrm{e}_{2}, \mathrm{e}_{3}, \mathrm{~h}_{1}$ and $\mathrm{h}_{2}$, as shown in the figure. A separate measurement on a piece of substrate (after superlattice layer was mechanically removed) at the same temperature shows that four of those carriers are still present (Fig. 3) but $\mathrm{e}_{1}$ is absent. This observation prompts us to conclude that $\mathrm{e}_{1}$ comes from the superlattice while the rest emanate from the substrate. In order to understand the genesis of $\mathrm{e}_{1}$,

we have estimated the energy barrier involved in its activation using variable temperature measurements. Its sheet carrier density was estimated (Eq. 1) and plotted as a function of inverse temperature, as shown in Fig. 4. From the slope of the plot in the temperature region close to the ambient, we have estimated the activation energy associated with $\mathrm{e}_{1}$ to be about $0.27 \mathrm{eV}$, in reasonable agreement with theoretical picture of superlattice band gap [8]. Moreover, ambient temperature photoluminescence measurements on an identical superlattice sample show a distinct peak in the same energy range, thus validating the main inference of QMSA. Further, excitation of $\mathrm{e}_{1}$ electrons across the superlattice band gap should result in creation of a low mobility hole, making an additional contribution to conduction within superlattice. We see a signature of this additional hole conductivity in terms of enhanced density of $h_{1}$ for $T>250 \mathrm{~K}$. 
Mobility of electron specie $\mathrm{e}_{1}$, estimated using Eq. 1, is plotted against temperature in Fig. 5. A steeper drop at low temperatures is a result of ionized impurity scattering [9], while gradual decrease at higher temperatures $(\mathrm{T}>100 \mathrm{~K})$ is due to phonon scattering [10]. We have noted that $\mu_{Q M S A} \sim \mathrm{T}^{-1.31}$, in good agreement with the exponent "-1.4" reported earlier [10]. Moreover, ambient temperature value of mobility obtained from present measurements $\left(\sim 10^{4} \mathrm{~cm}^{2} / \mathrm{Vs}\right)$ matches with that reported in literature [11].

Thus, results obtained by QMSA clearly show that electrical conduction within GaSb/InAs superlattice is linked to thermal excitation of carriers across a band gap of $0.27 \mathrm{eV}$.

\section{Conclusion:}

In conclusion, the nature of electrical transport in $\mathrm{GaSb} / \mathrm{InAs}$ superlattice was studied using variable magnetic field and variable temperature measurements. Mobility spectrum analysis shows that sample conductivity is dominated by dopants and impurities in GaSb substrate. However, it was possible to discern superlattice contribution due to the efficacy of the method. A lone electron specie associated with superlattice band gap $(0.27 \mathrm{eV})$ was noted in the temperature window close to the ambient, with a mobility of $\sim 10^{4} \mathrm{~cm}^{2} / \mathrm{Vs}$ at $300 \mathrm{~K}$.

It is also important to note that the accuracy of the parameters extracted using QMSA strongly depends on the contribution of superlattice to the total conduction. In order to improve this ratio thinner substrate wafers should be considered for GaSb/InAs superlattice growth. 


\section{References:}

[1] E. Plis, S. Annamalai, K. T. Posani, S. Krishna, R. A. Rupani, and S. Ghosh, J. Appl. Phys. 100, 014510/1-4 (2006).

[2] Andrew Hood, Darin Hoffman, Yajun Wei, Frank Fuchs, and Manijeh Razeghi, Appl. Phys. Lett. 88, 052112/1-3 (2006) and references therein.

[3] A. Rogalski and P. Martyniuk, Infrared Phys. Technol. 48, 39 (2006).

[4] H. Mohseni, M. Razeghi, G. J. Brown, and Y. S. Park, Appl. Phys. Lett. 78, 2107 (2001).

[5] J. Antoszewski and L. Faraone, Opto-Electon. Rev. 12, 347 (2004) and references therein.

[6] I. Vurgaftman, J. R. Meyer, C. A. Hoffman, D. Redfern, J. Antoszewski, L. Faraone and J. R. Lindemuth, J. Appl. Phys. 84, 4966 (1998).

[7] H.J. Haugan, G.J. Brown, F. Szmulowicz, L. Grazulis, W.C. Mitchel, S. Elhamri and W.D. Mitchell, J. Crystal Growth 278, 198 (2005)

[8] F. Szmulowicz, H. Haugan, and G. J. Brown, Phys. Rev. B 69, 155321/1-17 (2004).

[9] Joran H. Roslund, Ken Saito, Kyoichi Suzuki, Hiroshi Yamaguchi, Yoshiro Hirayama, Jpn. J. Appl. Phys. 39, 2448 (2000).

[10] P. S. Dutta, V. Prasad, H. L. Bhat and Vikram Kumar, J. Appl. Phys. 80, 2847 (1996).

[11] L. Bürkle, F. Fuchs, J. Schmitz, and W. Pletschen, Appl. Phys. Lett. 77, 1659 (2000). 


\section{Figure Captions:}

Fig. 1: Metal contacts at the corners of a $3 \mu \mathrm{m}$ thick superlattice layer, in Van der Pauw configuration. Thickness of GaSb substrate is about $490 \mu \mathrm{m}$.

Fig. 2 : A typical mobility spectrum of $\mathrm{GaSb} / \mathrm{InAs} / \mathrm{GaSb}$ superlattice sample, obtained by QMSA procedures, at $100 \mathrm{~K}$. A total of five carrier species are noted: three electrons $\mathrm{e}_{1}, \mathrm{e}_{2}$ and $\mathrm{e}_{3}$ (solid circles) and two holes $\mathrm{h}_{1}$ and $\mathrm{h}_{2}$ (open squares).

Fig. 3: Mobility spectrum of GaSb substrate at $100 \mathrm{~K}$. Note the absence of carrier $\mathrm{e}_{1}$.

Fig. 4: Sheet carrier density of $\mathrm{e}_{1}$ estimated using QMSA, shown as a function of inverse temperature. Band gap is estimated by a linear fit to the data in the range $260 \mathrm{~K}-300 \mathrm{~K}$.

Fig. 5: Temperature dependence of the mobility of $\mathrm{e}_{1}$, estimated using QMSA. 
Fig. 1

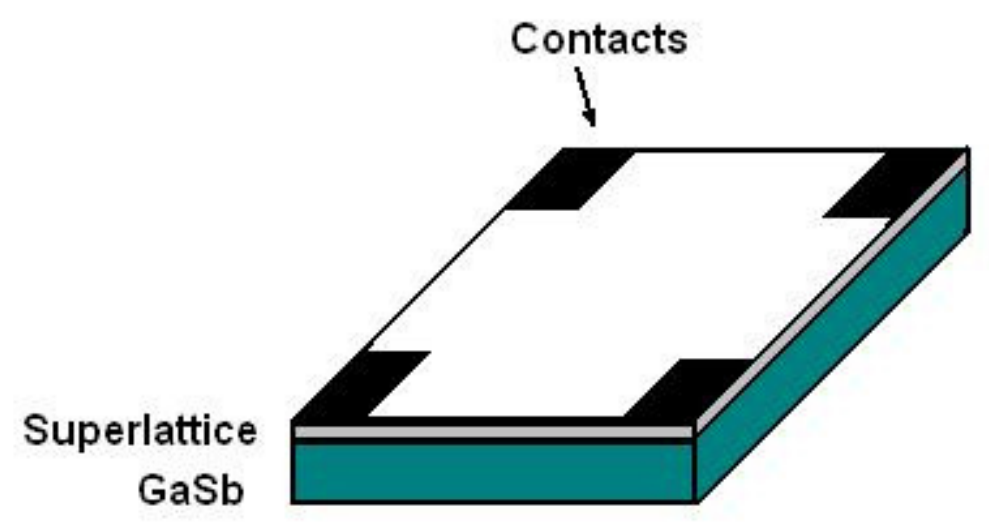


Fig. 2

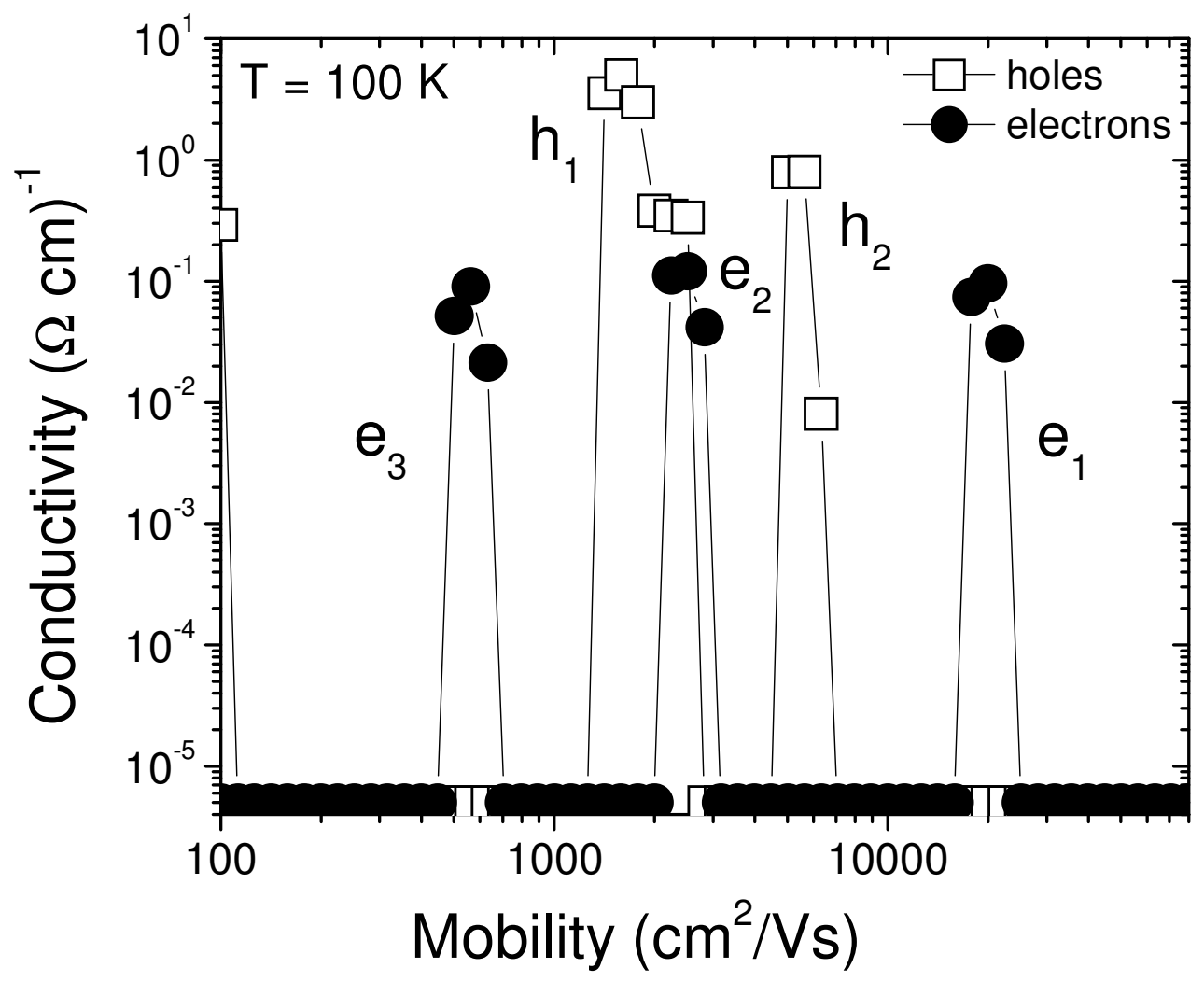


Fig. 3

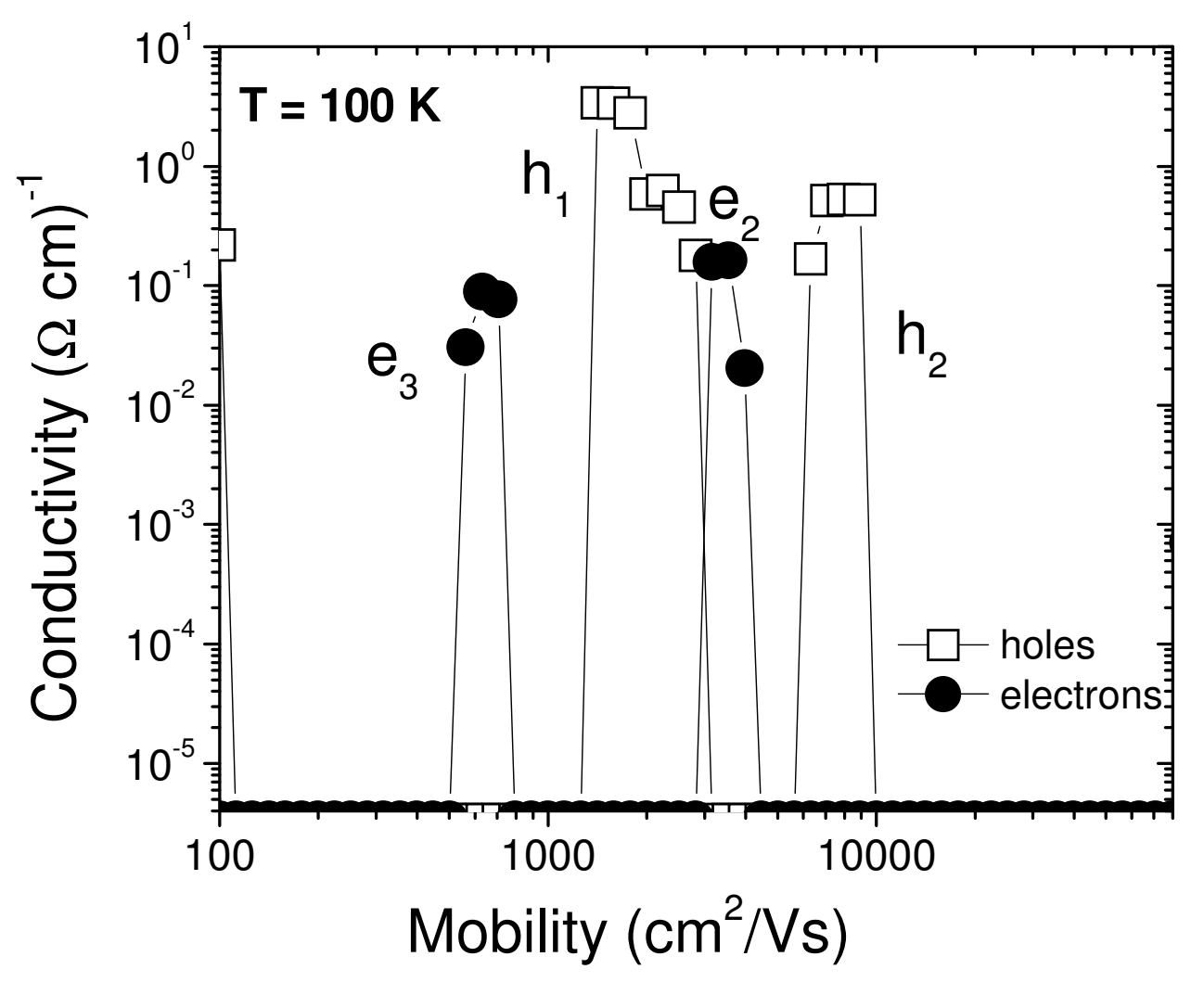


Fig. 4

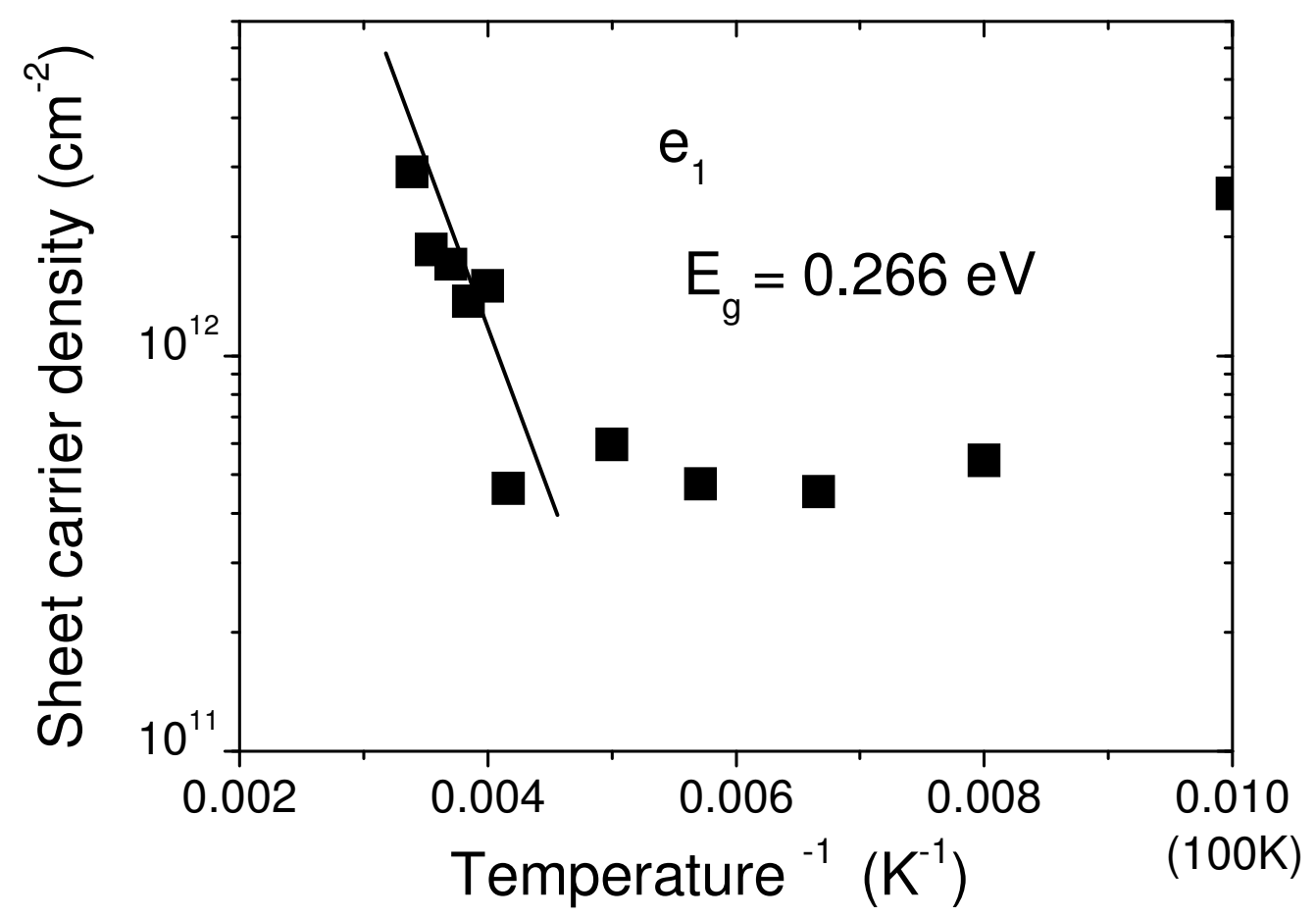


Fig. 5

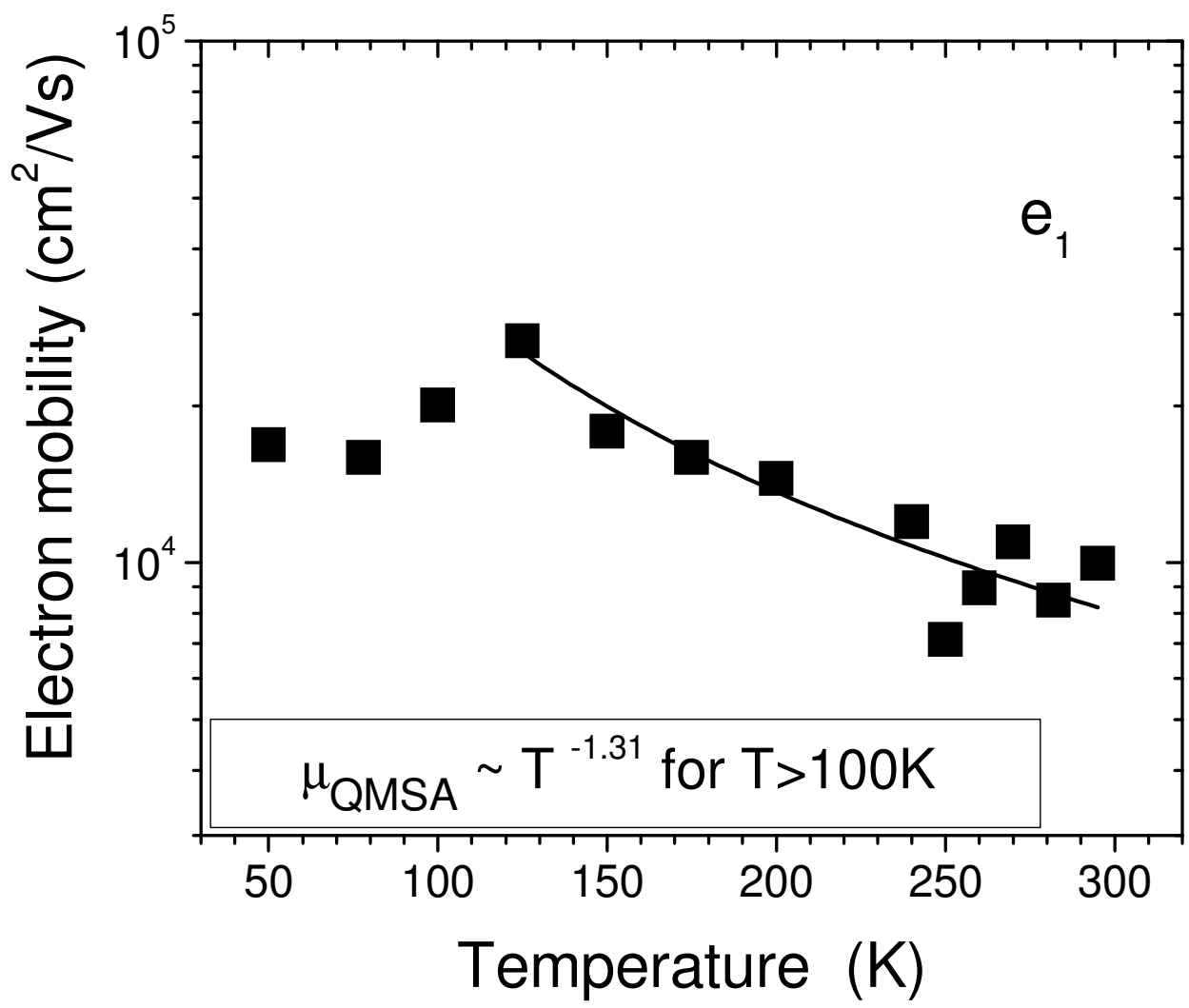

